ARTICLE

https://doi.org/10.1038/s41467-019-09544-9

\title{
D-Serine made by serine racemase in Drosophila intestine plays a physiological role in sleep
}

Xihuimin Dai (1) ${ }^{1,5}$, Enxing Zhou (10) 2,5, Wei Yang ${ }^{1}$, Xiaohui Zhang ${ }^{3}$, Wenxia Zhang ${ }^{1} \&$ Yi Rao ${ }^{1,4}$

Natural D-serine (D-Ser) has been detected in animals more than two decades ago, but little is known about the physiological functions of D-Ser. Here we reveal sleep regulation by endogenous D-Ser. Sleep was decreased in mutants defective in D-Ser synthesis or its receptor the N-methyl-D-aspartic receptor 1 (NMDAR1), but increased in mutants defective in D-Ser degradation. D-Ser but not L-Ser rescued the phenotype of mutants lacking serine racemase (SR), the key enzyme for D-Ser synthesis. Pharmacological and triple gene knockout experiments indicate that D-Ser functions upstream of NMDAR1. Expression of SR was detected in both the nervous system and the intestines. Strikingly, reintroduction of SR into specific intestinal epithelial cells rescued the sleep phenotype of $s r$ mutants. Our results have established a novel physiological function for endogenous D-Ser and a surprising role for intestinal cells.

\footnotetext{
${ }^{1}$ PKU-IDG/McGovern Institute for Brain Research, Peking University, Beijing 100871, China. ${ }^{2}$ College of Life Sciences, Beijing Normal University, Beijing 100875, China. ${ }^{3}$ State Key Laboratory of Natural and Biomimetic Drugs, Peking University, Beijing 100191, China. ${ }^{4}$ Chinese Institute for Brain Research Beijing 102206, China. ${ }^{5}$ These authors contributed equally: Xihuimin Dai, Enxing Zhou. Correspondence and requests for materials should be addressed to Y.R. (email: yrao@pku.edu.cn)
} 
$\Lambda$ mino acids exist in stereoisomers, with all common amino acids except glycine having L- and D-enantiomers depending on the relative spatial arrangement surrounding the a-carbon. Though L-amino acids were traditionally thought to be the only natural form, D-amino acids have been found in biological organisms. Free D-serine (D-Ser) has been found in species ranging from bacteria to mammals ${ }^{1-4}$. D-Ser is an effective co-agonist of the $\mathrm{N}$-methyl-D-aspartate subtype of glutamate receptor (NMDAR) ${ }^{5,6}$. D-Ser is synthesized from L-Ser by serine racemase (SR ${ }^{7}$ and degraded by $\mathrm{D}$-amino acid oxidase $(\mathrm{DAAO})^{4}$ and $\mathrm{SR}^{8}$. Distribution of D-Ser and NMDAR as determined by chemical measurement ${ }^{9}$ and immunohistochemistry ${ }^{10}$ supports D-Ser as an endogenous coagonist acting on the glycine modulatory site of the NR1 subunits of the NMDAR ${ }^{11,12}$. A role for endogenous D-Ser in synaptic transmission was confirmed by selective degradation of D-Ser with DAAO which attenuated NMDAR function and its rescue by D-Ser ${ }^{13}$. It was proposed that the synaptic NMDAR is activated by D-Ser, whereas the extrasynaptic NMDAR is gated by glycine ${ }^{14}$.

Sleep is important for animals and is regulated by both circadian and homeostatic processes ${ }^{15}$. While significant progress has been made in the molecular understanding of circadian rhythm, much less is known about homeostatic regulation of sleep. For more than a decade, Drosophila has been used as a model for genetic studies of sleep ${ }^{16,17}$. Genes and brain regions regulating sleep have been identified ${ }^{18-21}$.

Recently, NMDAR and D-Ser have been indicated to participate in sleep regulation in both flies and mammals ${ }^{22-24}$. However, whether D-Ser regulates sleep remains unclear. Here, through a genetic screen followed by a thorough investigation of the synthases, the oxidases, and the receptor of D-Ser, combined with pharmacological genetic epistasis experiments, we report evidence that sleep is regulated by D-Ser through NMDAR1. Furthermore, the synthases, the oxidases, and the receptor of D-Ser have all been found to be expressed in the central nervous system and in the intestine. Strikingly, the intestinal but not neuronal expression has been proved to be important for sleep regulation, indicating a novel role of the intestine in sleep regulation. Taken together, these results suggest that D-Ser made by intestinal SR promotes sleep through NMDAR1 in Drosophila.

\section{Results}

Decreased sleep in shmt mutants and rescue by L-Ser or D-Ser. In a screen of homozygous P-element insertion lines for mutations affecting sleep, we found that sleep duration was decreased when a P element was inserted into the CG3011 gene. Analysis of its sequence (Fig. 1a and Supplementary Fig. 1) indicates that CG3011 encodes the serine hydroxymethyltransferase (SHMT), which participates in the synthesis of L-Ser ${ }^{25,26}$ (Fig. 1b). There are three isoforms of shmt in fly, the original mutant uncovered by our screen contained a $\mathrm{P}$ element insertion in the $5^{\prime}$ noncoding region of isoform A (Fig. 1a). To investigate the function of Drosophila SHMT, we generated mutations in the shmt gene by using CRISPR-Cas9. Deletion of all three isoforms caused lethality, whereas frameshift mutations introducing a STOP codon in

a

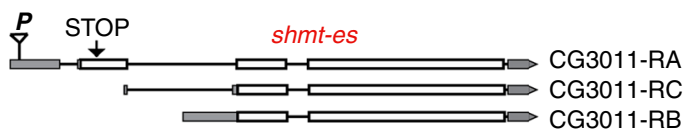

wt MQRARSTLTQKLRFCLSRDLNTKVGNPVNFETGKLSGALTRIAA shmt-es MQRARSTLTQKLRFCLSRDLNTSWQPG*

b
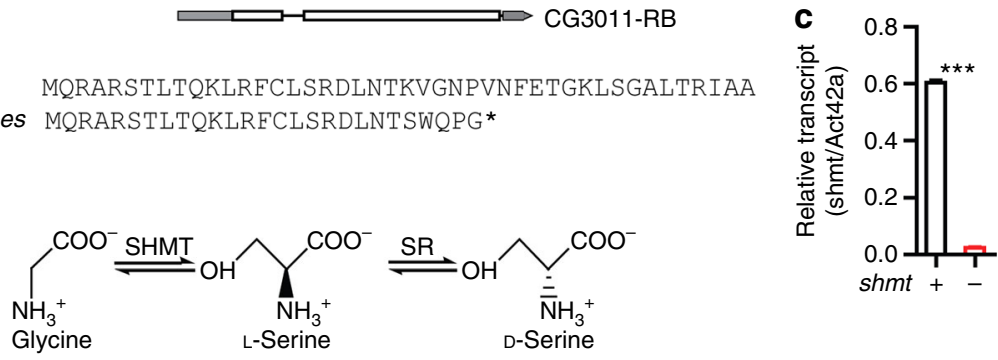

d
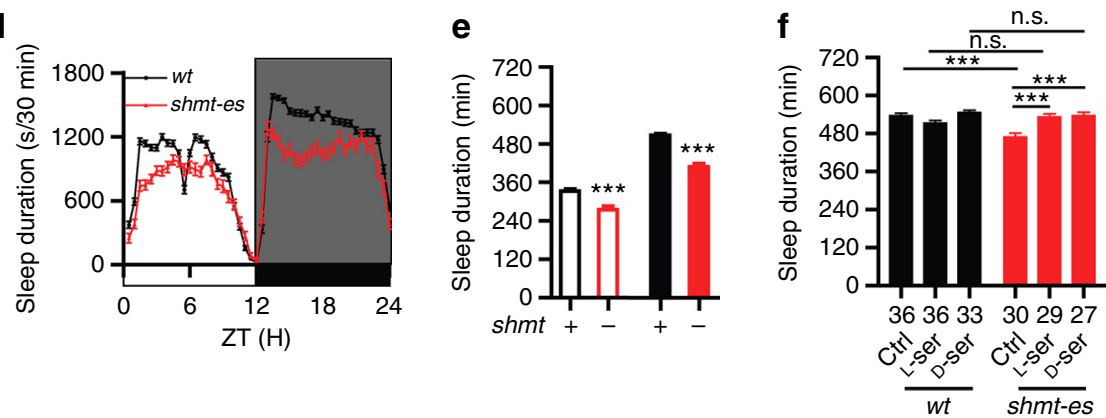

Fig. 1 Sleep phenotypes of shmt mutants. a A schematic representation of a point mutation leading to a premature stop codon in shmt (thus shmt-early stop or shmt-es). Also shown is the amino acid sequences of the shmt-es mutant line used here. Single gRNA generated insertion and/or deletion (indel) in the shmt gene, introducing a frameshift and a stop codon (asterisk). b A diagram of D-Ser synthesis pathway. c mRNA level of isoform A shmt in shmt-es was significantly reduced. $\mathbf{d}$ Sleep profiles of shmt-es (red) $(n=57)$ and wt (black) $(n=236)$ flies, plotted in 30 min bins. White background indicates the light phase (ZT 0-12); shaded background indicates the dark phase (ZT 12-24). e Statistical analyses. Daytime and nighttime sleep durations were significantly reduced in shmt-es flies. In this and other figures, open bars denote daytime sleep and filled bars nighttime sleep. $\mathbf{f}$ Drug treatment of both L- and D-Ser rescued the nighttime sleep duration of shmt-es flies to the $w t$ level. The number of flies used in the experiment was denoted under each bar. ${ }^{\star \star \star} P<0.001$, n.s. $P>0.05$, Mann-Whitney test was used in (c, e), two-way ANOVA test with Bonferroni posttests was used in (f) to compare the sleep durations between wt and shmt-es, Kruskal-Wallis test with Dunn's posttest was used in (f) to compare the sleep durations of shmt-es under different drug treatments. Error bars represent s.e.m. Male flies were used 
the first coding exon of shmt affecting only isoform A resulted in viable shmt mutants (shmt-es in Fig. 1a). The mRNA level of isoform A shmt in shmt-es was significantly decreased compared with wild type $(w t)$ flies detected by quantitative polymerase chain reaction (qPCR) analyses (Fig. 1c). The shmt-es mutants were backcrossed into an isogenic Canton-S (CS) line in our $\mathrm{lab}^{27}$, and used in further analysis.

Sleep was measured in $w t$ and shmt-es flies by video recording and analysis. When tested under the $12 \mathrm{~h}$ light/12 h dark (LD) condition, durations of both nighttime sleep and daytime sleep were significantly decreased in shmt-es flies (Fig. 1d, e). Because $\mathrm{L}$-Ser is the substrate for D-Ser synthesis (Fig. $1 \mathrm{~b})^{7}$, we tested whether the sleep phenotype of shmt mutants was attributed to Lor D-Ser by rescuing shmt mutants with either L-Ser or D-Ser. After eclosion, flies were raised with either sucrose or sucrose supplemented with L-Ser or D-Ser for 3 days before being transferred into recording tubes with the same media. Feeding either L-Ser or D-Ser could rescue the sleep defect of shmt-es flies (Fig. 1f). Thus, the sleep defect of shmt-es flies could be due to the lack of either L- or D-Ser.

Decreased sleep and increased arousal in $s r$ mutants. SR is responsible for D-Ser production in vivo ${ }^{28-30}$. Drosophila SR is encoded by CG8129 (Supplementary Fig. 2) ${ }^{31}$. To investigate the function of D-Ser, we generated sr knock-out (srko) flies in which most of the coding region of $s r$ was deleted (Fig. 2a). Under LD condition, the nighttime sleep duration was significantly reduced in srko flies (Fig. 2b, c). We also generated four other $s r$ mutants, including two deletion mutants sr-middle and sr-long (Supplementary Fig. 3a), and two insertion mutants SRKO-Gal4 and SRKO-Flp with the coding region replaced by the yeast Gal4 or Flp gene (Supplementary Fig. 3b). The duration of nighttime sleep but not that of daytime sleep was also reduced in these four mutants (Supplementary Fig. 3c-f). Because the nighttime sleep duration was decreased in all five $s r$ mutants as well as the shmt-es mutants, we thereafter focused on the role of D-Ser in nighttime sleep, but not the daytime sleep which was observed in only the shmt-es mutants but none of the five sr deletion mutants.

While sleep duration can directly reflect sleep defect, stimuliinduced arousal rate can reflect sleep intensity. Previous studies have shown that sleep duration and arousal can be regulated separately ${ }^{32,33}$. We tested arousal response to light in $w t$ and srko flies using similar method as previous studies ${ }^{34}$. On the 4 th night after being transferred to the recording tubes, sleeping flies were shined with light pulses for $1 \mathrm{~s}$ at zeitgeber time (ZT) 16, and then the numbers of flies that were awaken and that kept sleep were counted. The arousal rate was significantly elevated in srko flies under stimulus (Fig. 2e).

Latency to sleep was increased in srko flies whereas circadian rhythm and sleep recovery after sleep deprivation were not significantly different between srko and wt flies (Supplementary Fig. 4). Taken together, these results indicate that $s r$ is required

a

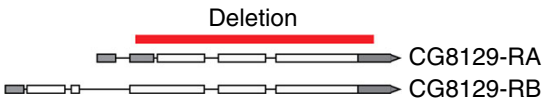

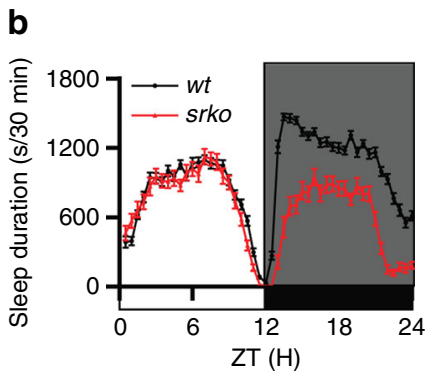

C

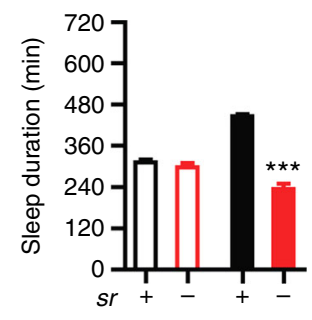

f 80

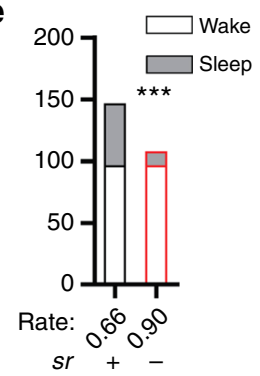

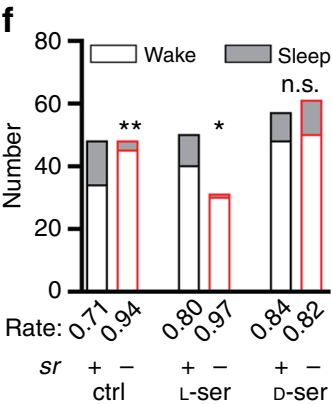

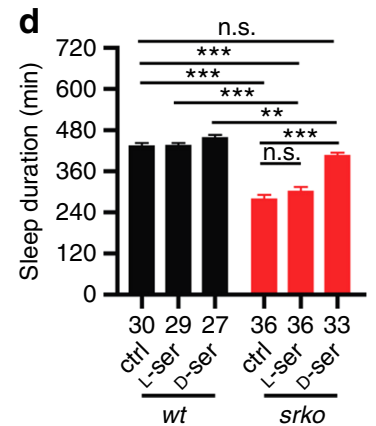

Fig. 2 Sleep phenotype of sr mutants. a A schematic representation of the CG8129 gene with the red bar indicating the region deleted in srko mutants. Two transcription variants (NM_141629, NM_169273) generate two proteins of 469aa (NP_649886) and 316aa (NP_731340), respectively. They have an identical C-terminal part while the longer variant has additional 153aa at the N-terminal region. In srko, aa 114-469 in the longer form and aa 1-316 in the shorter form were deleted. b Sleep profiles of srko (red) $(n=42)$ and wt (black) $(n=69)$ flies, plotted in 30 min bins. c Statistical analyses. Nighttime sleep durations were significantly reduced in shmt-es flies. $\mathbf{d}$ Drug treatment of D-Ser, but not L-Ser, rescued the nighttime sleep duration of srko flies to wt flies fed with mock. The number of flies used in the experiment was denoted under each bar. e Arousal rates of srko and wt flies under light stimuli. The arousal rate of srko flies was significantly increased. Numbers of flies that were aroused by the stimuli (open bars) and that kept sleep (filled bars) were plotted. Light stimuli were applied to wt and mutant flies as indicated. Arousal rate was denoted under each bar. $\mathbf{f}$ Drug treatment of D-Ser, but not L-Ser, rescued the arousal rate of srko flies to the wt level. ${ }^{\star \star \star} P<0.001,{ }^{\star \star} P<0.01,{ }^{\star} P<0.05$, n.s. $P>0.05$. Mann-Whitney test was used in (c), two-way ANOVA test with Bonferroni posttests was used in (d) to compare the sleep durations between wt and srko under the same treatment, Kruskal-Wallis test with Dunn's posttest was used in (d) for other statistical analyses. Fisher's exact test was used in (e, f). Error bars represent s.e.m. Male flies were used 
for regulation of nighttime sleep and stimulus-induced arousal response.

Role of D-Ser in sleep and arousal. SR is the only known enzyme responsible for D-Ser synthesis in vivo (Fig. 1b) ${ }^{35}$, L-Ser and DSer could be converted reciprocally to each other by SR. Sleep defect in shmt-es mutant flies is consistent with a role for either D- or L-Ser, whereas the phenotypes in srko mutants suggest that D-Ser is important for sleep. To further distinguish between Dand L-Ser, they were separately applied to srko flies.

As discussed earlier, both L-Ser and D-Ser could rescue the sleep defect of shmt-es flies (Fig. 1f). However, only D-Ser, but not L-Ser, could rescue the sleep defect of srko flies (Fig. 2d). No significant sleep change was observed in srko flies fed with L-Ser compared to mock. By contrast, the nighttime sleep duration of srko was rescued by D-Ser to the level of $w t$ flies fed with mock. When we examined the arousal response, we found that the arousal rate of srko flies was also rescued to the $w t$ level by D-Ser, but not by L-Ser (Fig. 2f). These results suggest that D-Ser, but not L-Ser, is important for sleep and arousal.

Increased sleep and decreased arousal in daao-dko mutants. DSer is degraded by DAAOs (Fig. 3a). There are two genes encoding DAAO in Drosophila: CG12338 and CG11236 36 . To investigate their functional significance, we generated deletion mutants for each of the gene (Fig. 3b).

When a single daao was interrupted, there was not much change in sleep durations: nighttime sleep duration was significantly reduced in CG11236ko flies, while daytime sleep duration of CG11236ko and daytime and nighttime sleep durations of CG12338ko were not different from that in the $w t$ (Fig. 3c, d). In order to tell that if this is due to the redundant function of these two daao genes, we generated double knock-out (daao-dko) flies with both genes interrupted. We found that both daytime and nighttime sleep durations were significantly increased in daao-dko flies (Fig. $3 \mathrm{c}, \mathrm{d}$ ), and the arousal rate of daao-dko flies was significantly decreased (Fig. 3e). The opposite sleep and arousal phenotype of daao-dko flies to that in the srko flies further supports that D-Ser promotes sleep and inhibits the arousal response in Drosophila.

D-Ser regulation of sleep through NMDAR1. D-Ser is a coagonist of the NMDA receptor (NMDAR) ${ }^{13,14}$. There are two NMDAR subunits in Drosophila but only NMDAR1 contains the D-Ser binding site ${ }^{37}$. Pan-neuronal NMDAR1 knockdown by elav-Gal4 driven RNA interference (RNAi) reduces sleep in Drosophila ${ }^{22}$, but it did not distinguish whether the sleep effect was caused by D-Ser or by other NMDAR1 agonists, such as glycine. Recently, a study has found that NMDAR-mediated field excitatory post-synaptic potentials (NMDA-fEPSPs) and D-Ser levels fluctuate with sleep need in mice ${ }^{24}$, further raising the possibility of D-Ser regulating sleep through NMDAR1, in both flies and mammals.

To investigate whether NMDAR1 regulates sleep, we generated $n m$ darlko by replacing the first three coding exons of nmdar 1 with 2A-Gal4-STOP right after the start codon (Fig. 4a). Similar with srko flies, sleep duration was significantly decreased and arousal rate significantly increased in nmdarlko flies (Fig. $4 \mathrm{~b}-\mathrm{d}$ ).

We carried out two experiments to investigate the relation between D-Ser and NMDAR1 in sleep and arousal: pharmacological application of L-Ser and D-Ser on nmdar1ko flies, and a

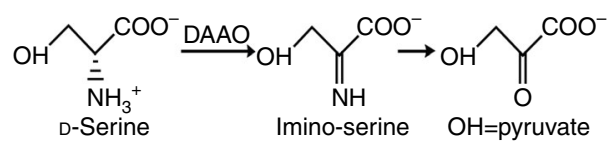

b
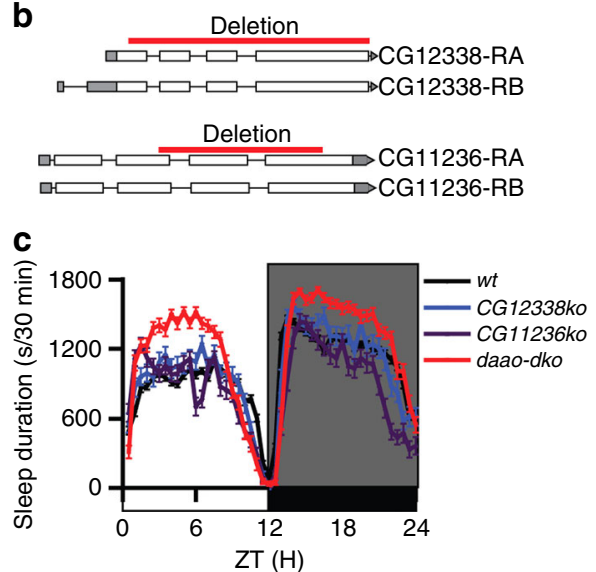

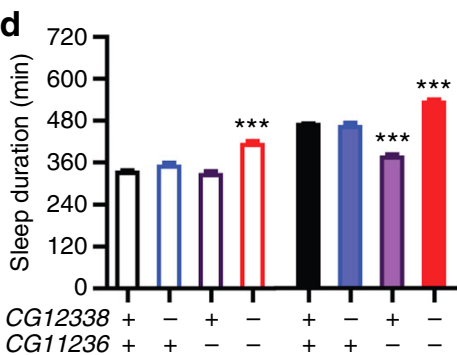

e

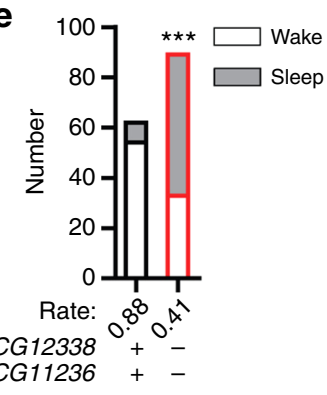

Fig. 3 Sleep phenotype of daao mutants. a A diagram of D-Ser degradation pathway. b Schematic representations of CG12338 and CG11236 genes. The red bars indicate regions deleted in mutant flies. For CG12338, two transcription variants (NM_001299363, NM_136759) generate the same protein of 335aa (NP_001286292, NP_610603) and most of the coding region except the first 97 base pairs (bp) was deleted in the CG12338 knock-out (cg12338ko) flies. For CG11236, two transcription variants (NM_135231, NM_001258985) generate two proteins of 341aa (NP_609075) and 338aa (NP_001245914), respectively. CG11236 knock-out (cg11236ko) contained the first 94aa because of deletion of 284 bp to 889 bp (NM_135231) or 284 bp to 880 bp

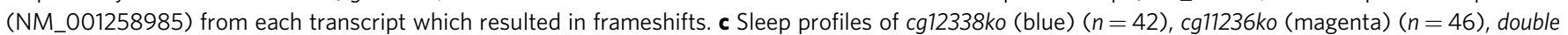

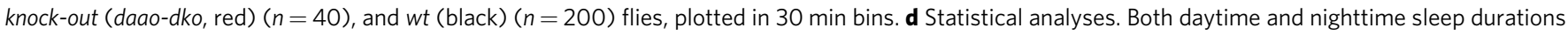
were significantly increased in daao-dko flies, nighttime sleep duration was significantly decreased in cg11236ko flies. e Arousal rate was significantly reduced in daao-dko flies. Numbers of flies were plotted for wt (black) and daao-dko (red) flies. ${ }^{\star \star \star} P<0.001$. Mann-Whitney test was used in (d), Fisher's exact test was used in (e). Error bars represent s.e.m. Male flies were used 

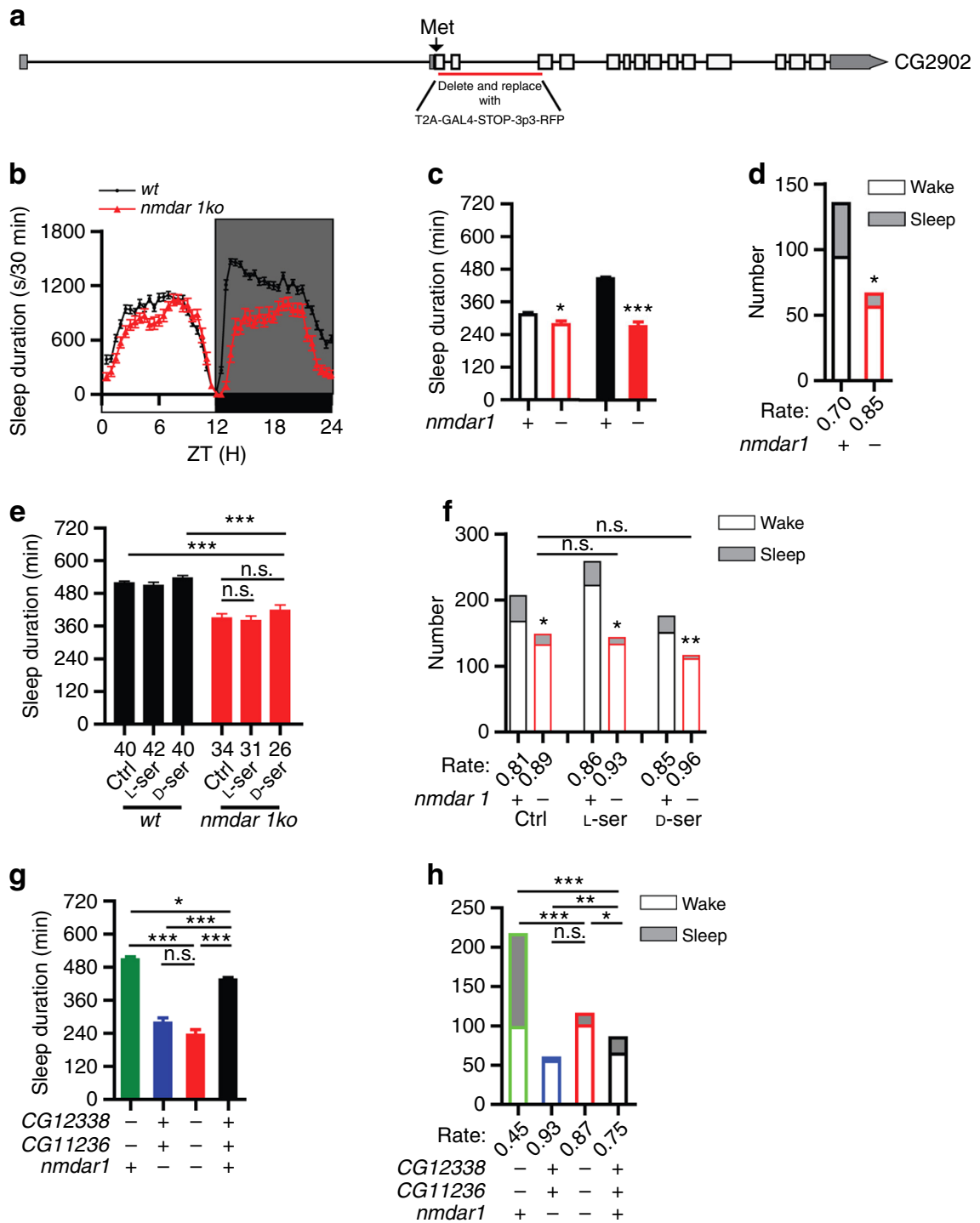

Fig. 4 Regulation of sleep by D-Ser upstream of the NMDAR1. a A schematic representation of nmdar1 gene. The single transcription variant (NM_169059) generates a protein of 997aa (NP_730940). The sequence from aa2 to 107 was deleted and replaced with T2A-Gal4-STOP-3P3-RFP in nmdar1ko flies. b Sleep profiles of nmdarlko (red) $(n=36)$ and wt (black) $(n=69)$ flies, plotted in 30 min bins. c Statistical analyses. Daytime and nighttime sleep durations were significantly reduced in nmdarlko flies. d Arousal rate of nmdarlko flies (red) was significantly higher than that of wt (black). Numbers of flies that were aroused (filled bars) and that kept sleep (open bars) were plotted for nmdarlko and wt flies. e, $\mathbf{f}$ Neither L- nor D-Ser affected the sleep duration (e) or the arousal rate (f) of nmdariko flies. Numbers below each bar represent the number of flies tested in (e). $\mathbf{g}, \mathbf{h}$ The sleep phenotype ( $\mathbf{g}$ ) and arousal phenotype (h) of daao-dko flies were masked by nmdarlko in triple knockout flies. Nighttime sleep durations were significantly increased in daao-dko (green) ( $n=46)$ flies, and significantly decreased in nmdar1ko (blue) $(n=32)$ and triple knockout (red) $(n=34)$ flies, compared to wt (black) ( $n=47)$ flies (g). Arousal rate was significantly decreased in daao-dko (green) flies, and significantly increased in nmdarlko (blue) and triple knockout (red) flies, compared to wt (black) flies (h). ${ }^{\star \star \star} P<0.001$, ${ }^{\star \star} P<0.01,{ }^{\star} P<0.05$, n.s. $P>0.05$. Mann-Whitney test was used in (c), two-way ANOVA test with Bonferroni posttests was used in (e) to compare the sleep durations between wt and nmdarlko under the same treatment, Kruskal-Wallis test with Dunn's posttest was used in (e) for other statistical analyses and in (g). Fisher's exact test was used in (d), (f), and (h). Error bars represent s.e.m. Male flies were used

generation of triple knock-out flies lacking the nmdarl and the two daao genes.

Although D-Ser rescued the sleep defect and arousal defect in shmt-es and srko flies (Figs. If and 2d, f), neither L-Ser nor D-Ser could affect the sleep duration and arousal rate of nmdarlko mutants (Fig. 4e, f). These results support that D-Ser lies downstream of SHMT and SR but upstream of NMDAR1 in sleep regulation.

To test the epistasis relationship of daao genes and the nmdar1 gene, triple knockout flies carrying all three mutations of CG12338ko, CG11236ko, and nmdarlko were generated by combining daao-dko and nmdarlko together. Triple knockout flies phenocopy $n m d a r 1 k o$ flies in sleep duration and arousal rate: sleep duration was increased and arousal rate decreased in daao-dko flies, while sleep duration was decreased and arousal rate increased in $n$ mdarlko and triple knockout flies (Fig. 4g, h). No significant difference was detected between the sleep duration and arousal rate of nmdarlko and triple knockout flies (Fig. $4 \mathrm{~g}, \mathrm{~h}$ ). These results indicate that nmdarl acts downstream of daao.

Thus, both the pharmacological experiment and the genetic epistasis experiment support that D-Ser functions through NMDAR1 to regulate sleep in Drosophila. 
Expression patterns of $\boldsymbol{s h m t}$, sr, daao, and nmdar1. To examine the expression of genes participated in the synthesis, degradation, and function of D-Ser, we fused Gal4 in-framely to shmt, sr, CG12338, CG11236, and nmdarl, making the shmt-KIGal4, SR-KIGal4, CG12338-KIGal4, CG11236-KIGal4, and nmdar1-KIGal4 lines (Supplementary Table 1). Then UAS-mCD8::GFP was driven by these lines to label the membrane of cells expressing them respectively.

We found that all five lines were expressed in the brain, the ventral nerve cord (VNC), and the gut (Fig. 5, Supplementary
Fig. 5). shmt-KIGal4 was expressed in the glia cell and neurons in the brain and the VNC (Fig. 5a, b), and in the midgut (Fig. 5c, Supplementary Fig. 5a). SR-KIGal4 was expressed in four neurons in the subesophageal ganglion (SOG) of the brain (Fig. 5d), in four pairs of neuronal tracts projecting to the prothoracic, mesothoracic, metathoracic neuromere $(\mathrm{PN}, \mathrm{MN}$, $\mathrm{MtN}$ ), and the abdominal center (AC) of the VNC (Fig. 5e), and in the midgut enterocytes (ECs) (Fig. 5f, Supplementary Fig. 5b).
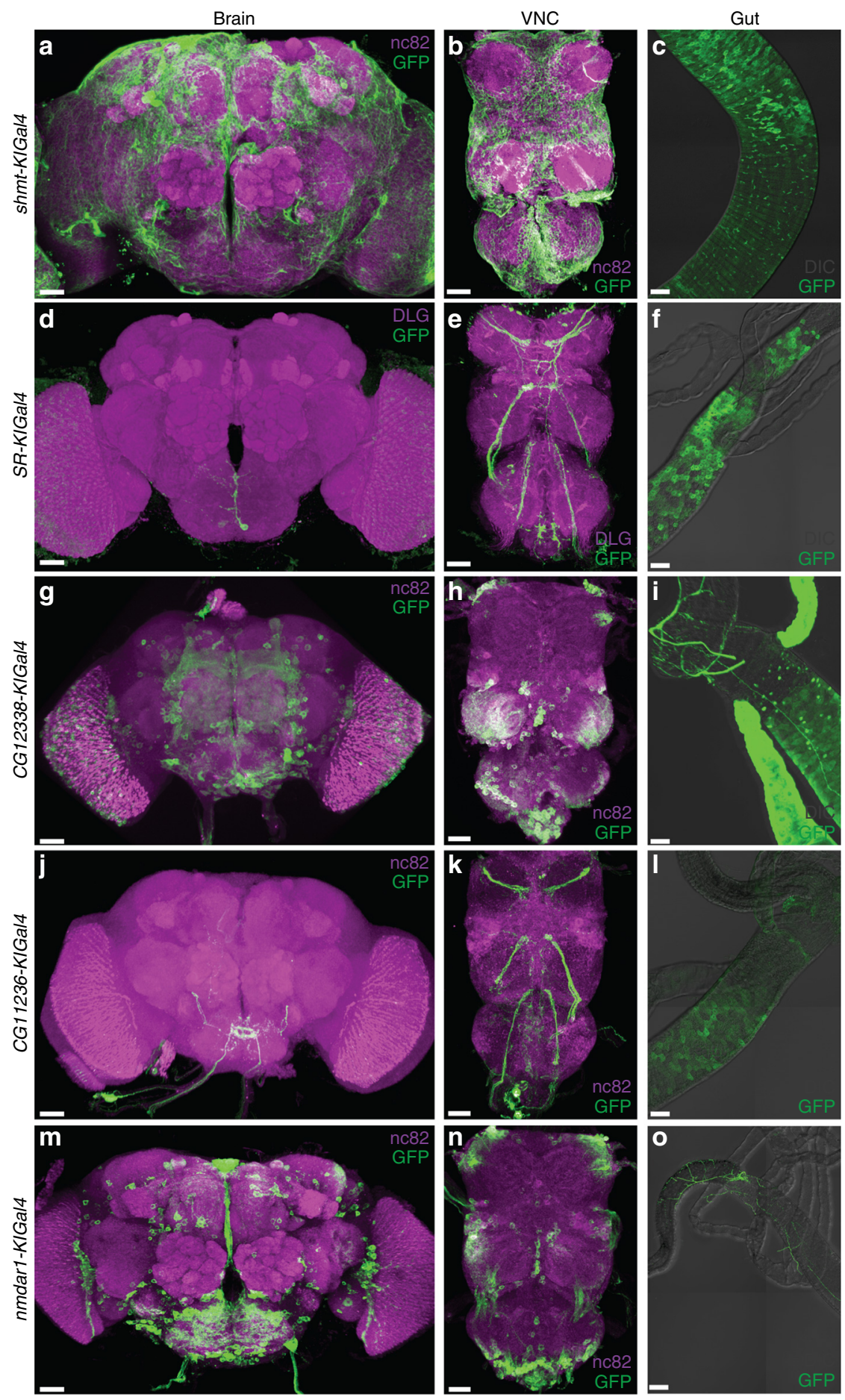

Fig. 5 Expression patterns of shmt, sr, daao, and nmdar1. Expression patterns of shmt-KIGal4 (a-c), SR-KIGal4 (d-f), CG12338-KIGal4 (g-i), CG11236-KIGal4

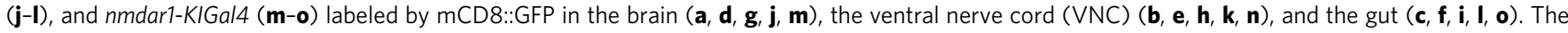
tissues were immunostained with anti-GFP and anti-DLG in (d, e), immunostained with anti-GFP in $(\mathbf{c}, \mathbf{f}, \mathbf{i}, \mathbf{I}, \mathbf{0})$, immunostained with anti-GFP and nc82 in other panels. Scale bars are $30 \mu \mathrm{m}$ 
CG12338-KIGal4 was expressed in the MB, the PI, and the SOG of the brain (Fig. $5 \mathrm{~g}$ ), in the $\mathrm{MN}$, the $\mathrm{MtN}$, and the $\mathrm{AC}$ of the VNC (Fig. 5h), and in the midgut, the Malpighian tubules, and the neurons projecting to the hindgut (Fig. 5i, Supplementary Fig. 5c). CG11236-KIGal4 was expressed similarly to SR-KIGal4: in quite a few neuron tracts projecting to the SOG of the brain (Fig. 5j), in four pairs of neuronal tracts projecting to the $\mathrm{PN}$, the $\mathrm{MN}$, the $\mathrm{MtN}$, and the $\mathrm{AC}$ of the $\mathrm{VNC}$ (Fig. 5k), and in the midgut ECs (Fig. 5l, Supplementary Fig. 5d).

nmdar1-KIGal4 was expressed broadly in the brain, including the PI, the SOG, the FSB, and the superior neuropils (SNP) (Fig. $5 \mathrm{~m}$ ), in the AC and the afferent neurons of the PN of the VNC (Fig. 5n), and in neurons projecting to the proventriculus, the midgut regions $\mathrm{R} 1$ and $\mathrm{R} 5$, and the hindgut (Fig. 5o, Supplementary Fig. 5e).

Intestinal SR in sleep regulation. Given that the synthases, the oxidases, and the receptor of D-Ser were all found to be expressed in the central nervous system and the gut, we next seek to identify which part is required for D-Ser to promote sleep by reintroducing UAS-SR into different parts in the srko background.

The nighttime sleep duration of $s r$ mutants was rescued by the reintroduction of UAS-SR back into $s r$-expressing cells (Fig. 6a) labeled by SRKO-Gal4 in which $2 A-G a l 4-S T O P$ was fused to the start codon of $s r$ (Supplementary Table 1). However, panneuronal expression of $s r$ driven by Elav-Gal4 failed to rescue the nighttime sleep duration (Fig. 6b), suggesting that $s r$ does not function in neurons to promote sleep. Furthermore, we labeled non-neuronal sr-expressing cells by SRKO-Gal4, ElavGal80, in which neuronal expression but not intestinal expression of SRKO-Gal4 was blocked by Elav-Gal80 (Fig. 6g-i). Reintroduction of $s r$ back into non-neuronal $s r$-expressing cells also rescued the sleep defect of $s r$ mutants (Fig. 6j), suggesting that neuronal $s r$ is not necessary for sleep promoting. Taken together, these results suggest that neuronal $s r$ is neither sufficient nor necessary for sleep promoting, thus $s r$ functions elsewhere to regulate sleep.

Because $s r$ was expressed in the midgut ECs (Fig. 5f, Supplementary Fig. 5b), we used MyoIA-Gal4 which was known to drive GFP expression in midgut $\mathrm{ECs}^{38}$ to test the role of intestinal $\mathrm{SR}$ in sleep regulation. $\mathrm{mCD} 8:$ GFP was driven by MyoIA-Gal4 to label MyoIA-expressing cells, MyoIA-Gal4 was expressed in the midgut ECs (Fig. $6 \mathrm{~m}$ ) as well as in the neurons in the brain and the VNC (Fig. 6k, 1), while no expression of MyoIAGal4 was found in the genital and the internal surface of the abdominal cuticle which is covered by the fat body and the oenocytes (Supplementary Fig. 6). Colocalization of MyoIA and $s r$ was detected in the gut by simultaneously labeling $s r$-expressing cells with GFP and the nuclei of MyoIA-expressing cells with StingerRed (Fig. 6c-e). We also intersected MyoIA and $s r$ by expressing UAS-FRT-STOP-FRT-GFP in MyoIA-expressing cells, and expressing LexAop-Flp in sr-expressing cells. Thus, in cells that co-expressing MyoIA and $s r$, the stop cassette between the UAS and GFP was removed by the Flp recombinase, labeling the cells with GFP (Fig. 6f). Expression of $s r$ in MyoIA-expressing cells rescued the sleep defect of $s r$ mutants (Fig. 6n). We also used Elav-Gal80 to block the neuronal expression of MyoIA-Gal4 (Fig. $60-q$ ), and the nighttime sleep duration of $s r$ mutants was rescued by expression of $s r$ in non-neuronal MyoIA-expressing cells (Fig. 6r). Moreover, the sleep duration was significantly decreased and sleep latency significantly increased when $s r$ was knocked-down with RNAi specifically in the gut (Supplementary Fig. 7a, d) or daao was overexpressed specifically in the gut (Supplementary Fig. 7b, c, e, f) with daao cDNA driven by MyoIA-Gal4, Elav-Gal80. Taken together, these results support that SR expressed in intestinal cells is important for regulating sleep in Drosophila.

\section{Discussion}

Our findings have revealed both a novel function for D-Ser and a novel role for intestinal cells. Results from mutations of five genes (two genes required for D-Ser synthesis, two for D-Ser degradation, and one for the D-Ser receptor), taken together with those from the pharmacological application of L- and D-Ser, support the conclusion that $\mathrm{D}$-Ser plays an important role through NMDAR1 in regulating sleep in Drosophila. Furthermore, results from genetic rescue experiments with neuronal and intestinal drivers indicate that intestinal SR regulates sleep.

The evidence for D-Ser function in sleep is strong. Phenotypic analysis indicates that D-Ser is important for nighttime sleep and arousal. Nighttime sleep and arousal phenotypes of shmt, and $s r$ mutants are opposite to those of daao-dko mutants, consistent with roles of D-Ser in increasing sleep and decreasing arousal. Further support was provided by the finding that D-Ser could rescue the sleep and arousal phenotypes in shmt and sr mutants, whereas L-Ser was unable to rescue the sleep and arousal phenotypes in $s r$ mutants. In Drosophila, while the functional significance of D-Ser in sleep is clear, a role for L-Ser appears unlikely but cannot be completely ruled out at this point.

While NMDAR1 could affect circadian rhythm in mice ${ }^{39,40}$, it is surprising for a well-known excitatory receptor to promote sleep. Our results from nmdar1 knockout flies and $s r$ knockout flies provide the strongest in vivo evidence for an essential role of NMDAR1 in promoting sleep. These results are consistent with, but cleaner than, the previous RNAi results in flies ${ }^{22}$. NMDAR1 has recently been implicated in regulating sleep in flies ${ }^{22,23}$ : panneuronal knocked down of NMDAR1 or NMDAR2 through RNAi or feeding of the NMDAR antagonist MK801 to flies reduced sleep duration ${ }^{22}$. So far, regional RNAi had failed to reveal specific regions where NMDAR1 regulates sleep ${ }^{22}$. NMDAR1 expression has been detected in the R2 ring of the EB which is important for sleep homeostasis ${ }^{23}$, though it remains unknown whether NMDAR1 in the $\mathrm{R} 2$ ring regulates sleep.

Roles for D-Ser in regulating mammalian sleep and arousal remain to be investigated. The saturation level of the glycine binding site in the NMDAR1 correlates with sleep need in mice 24 Total serine level increased to $\sim 487 \%$ during slow wave sleep (SWS) in the ventrolateral posterior nuclei (VLPN) of the cat thalamus ${ }^{41}$. D-Ser reduces sedative response induced by alcohol in flies and rodents ${ }^{31,42}$. A report of the effect of daao ablation on promoting sedative response in mice under novel environment ${ }^{43}$ was refuted by further analysis ${ }^{44}$. Because D-Ser was only increased in some but not all regions in the brain after the elimination of a single daao ${ }^{45}$, the function of D-Ser in mammalian sleep remains unclear.

Both glial and neuronal distribution of D-Ser and SR have been reported in mammals ${ }^{35,46}$. Early studies have detected D-Ser to be present in glia $10,47,48$. D-Ser has been considered as a major gliotransmitter ${ }^{49-51}$ and its release is triggered by non-NMDAR glutamate receptor ${ }^{48,49}$. However, SR and D-Ser were also found in neurons $47,52,53$. Both the neuronal and glial release of D-Ser have been detected ${ }^{54}$. Using conditional SR knocked out mice, the majority of SR (65\%) and extracellular D-Ser have been suggested to be of neuronal origin ${ }^{55}$. Our present study with Drosophila using SR-KIGal4 identified only neuronal but no glial expression of SR.

A striking finding here is that SR is not only expressed in the nervous system, but that it is expressed and functions in intestinal cells to regulate sleep in Drosophila. Through region-specific rescue, knock-down, and overexpression studies (Fig. 6, 

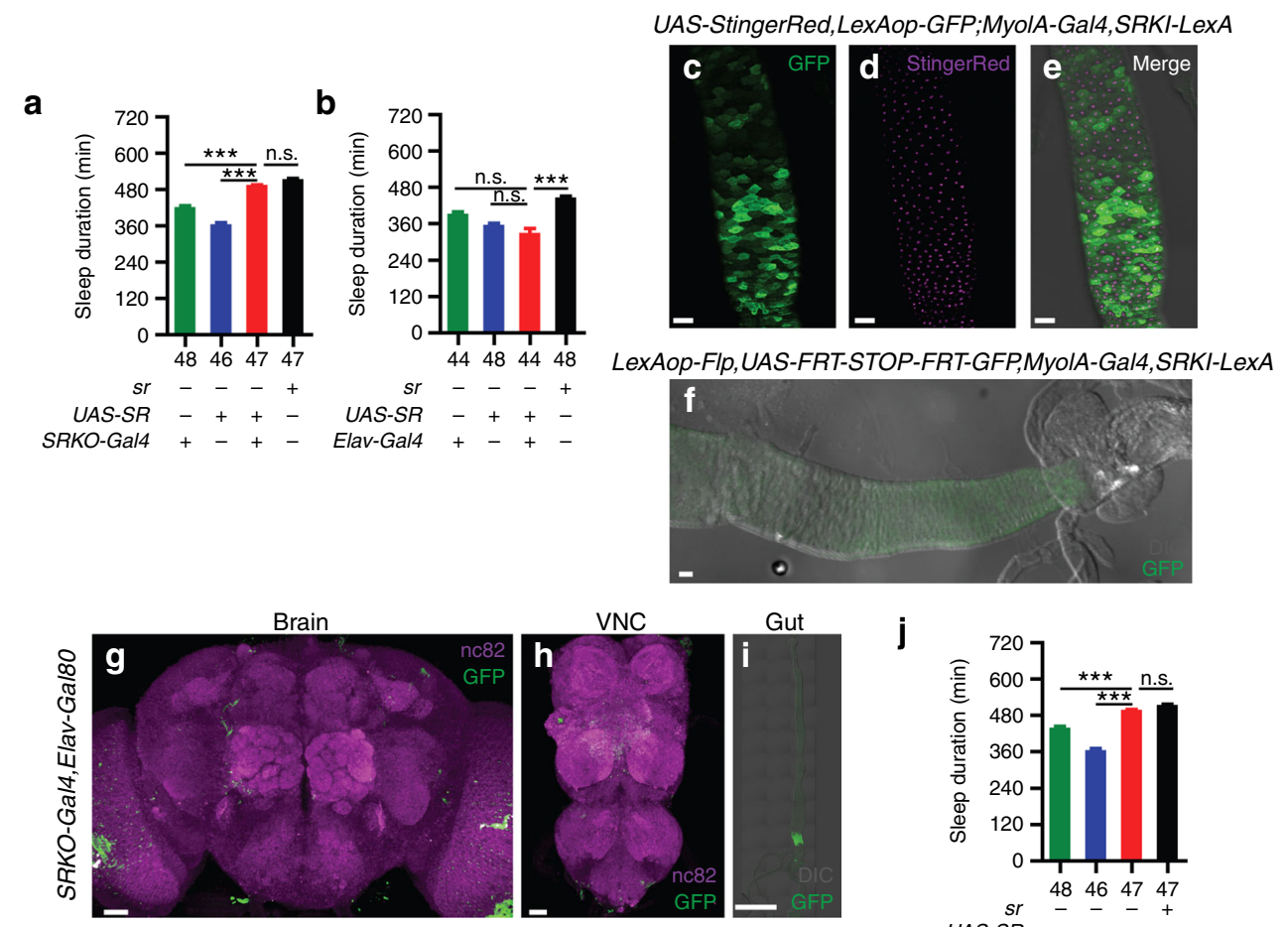

j
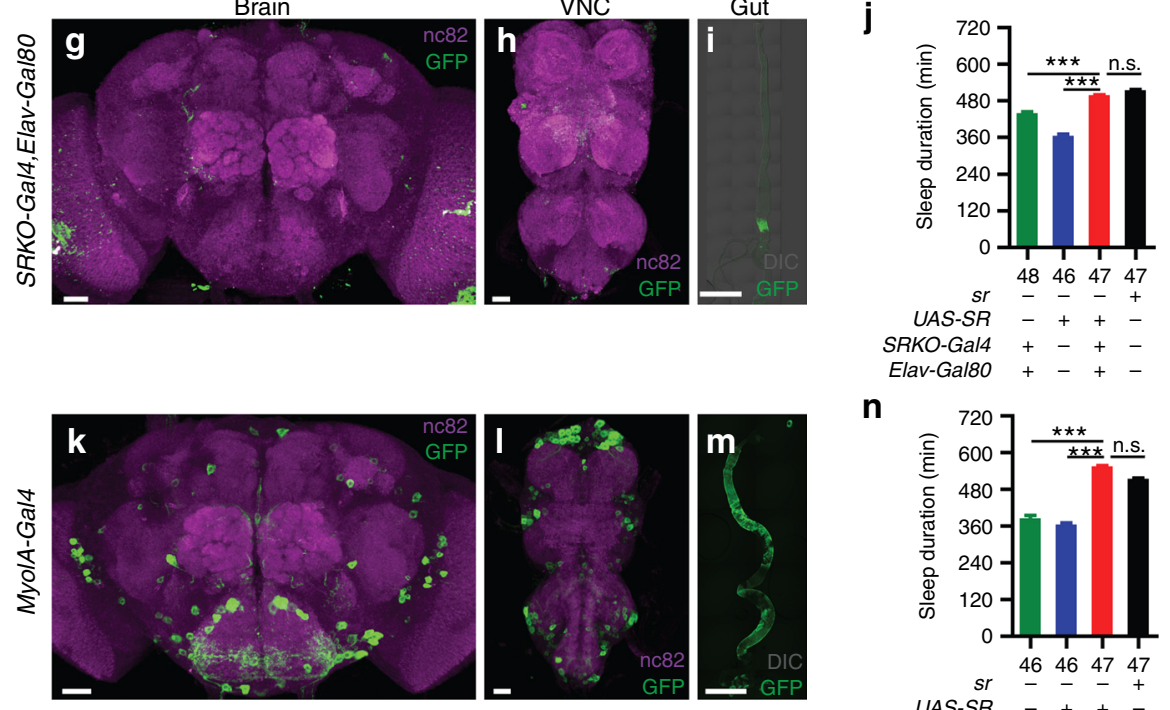

n
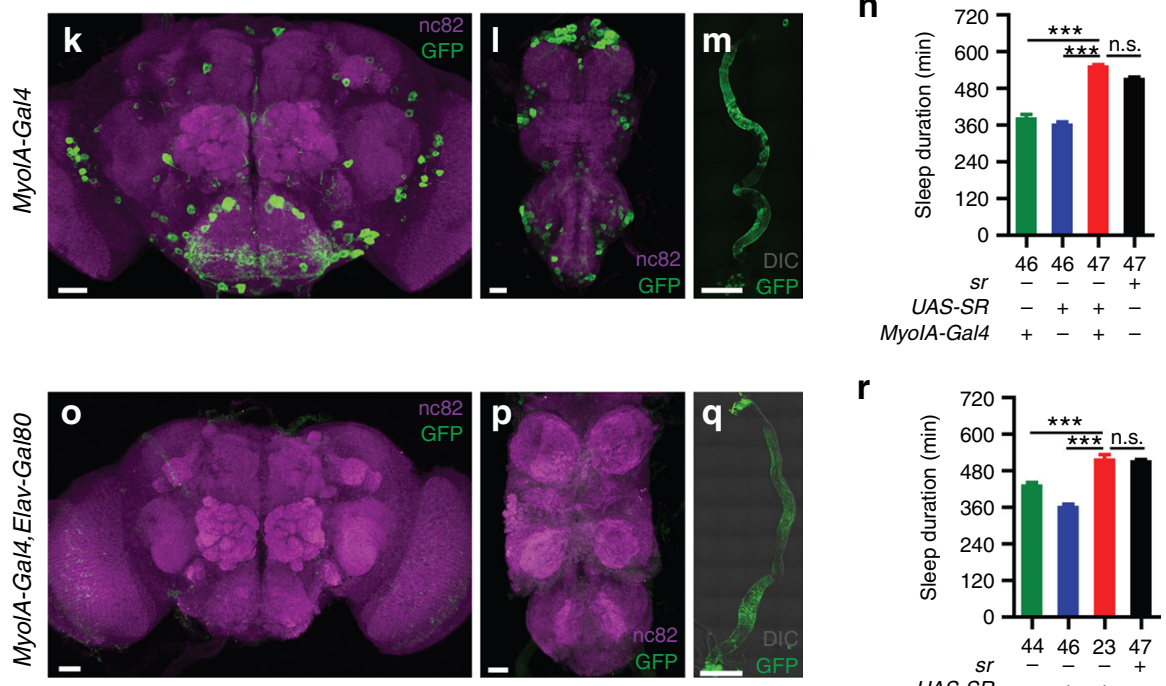

$\mathbf{r}$

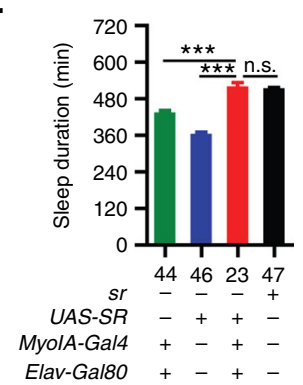

Fig. 6 Requirement of intestinal but not neural SR in sleep regulation. a Reintroduction of $s r$ in $s r$-expressing cells rescued the nighttime sleep defect of $s r$ mutants. Nighttime sleep durations of srko/SRKO-Gal4 (green), srko/srko,UAS-SR (blue), SRKO-Gal4/srko,UAS-SR (red), and wt (black) flies were plotted. b Reintroduction of $s r$ pan-neuronally failed to rescue the sleep defect of $s r$ mutants. Nighttime sleep durations of Elav-Gal4/Y; srko/srko (green), srko/srko, UAS-SR (blue), Elav-Gal4/Y; srko/srko,UAS-SR (red), and wt (black) flies were plotted. c-e sr-expressing cells and MyolA-expressing cells overlap in the fly gut. The nuclei of MyolA-expressing cells were labeled by StingerRed driven by MyolA-Gal4 (d), and the sr-expressing cells were labeled by GFP driven by SRKI-LexA (c). f Expression patterns of LexAop-Flp, UAS-FRT-STOP-FRT-GFP, MyolA-Gal4, SRKI-LexA flies in the gut. Cells co-expressing MyolA and sr were labeled with GFP. $\mathbf{g}$-i sr-expressing non-neuronal cells were labeled by SRKO-Gal4,Elav-Gal80 (i), whereas the neural cells expression was blocked ( $\mathbf{g}$, $\mathbf{h}$ ). $\mathbf{j}$ Reintroduction of $s r$ driven by SRKO-Gal4,Elav-Gal80 rescued the sleep defect of sr mutants. Nighttime sleep durations of srko/SRKO-Gal4,Elav-Gal80 (green), srko/srko,UAS-SR (blue), SRKO-Gal4,Elav-Gal80/srko,UAS-SR (red), and wt (black) flies were plotted. k-m Expression patterns of MyolA-Gal4 in the brain (k), the VNC (I), and the gut ( $\mathbf{m})$ labeled by mCD8::GFP. $\mathbf{n}$ Expression of $s r$ in MyolA-expressing cells rescued the nighttime sleep defect of sr mutants. Nighttime sleep durations of srko/srko,MyolA-Gal4 (green), srko/srko,UAS-SR (blue), srko,MyolA-Gal4/srko,UAS-SR (red), and wt (black) flies were plotted. o-q MyolAexpressing non-neuronal cells were labeled by MyolA-Gal4,Elav-Gal80 (q), whereas the neural cells expression was blocked (o, p). $\mathbf{r}$ Expression of sr driven by MyolA-Gal4,Elav-Gal80 rescued the sleep defect of sr mutants. Nighttime sleep durations of srko/srko,MyolA-Gal4,Elav-Gal80 (green), srko/srko,UAS-SR (blue), srko,MyolA-Gal4,Elav-Gal80/srko,UAS-SR (red), and wt (black) flies were plotted. The tissues were immunostained with anti-GFP in (c-f), (i, $\mathbf{m}$ ), and $(\mathbf{q})$, immunostained with anti-GFP and nc82 in $(\mathbf{g}, \mathbf{h}, \mathbf{k}, \mathbf{I}, \mathbf{0}, \mathbf{p})$. Scale bars are $500 \mu \mathrm{m}$ in $(\mathbf{i}, \mathbf{m}, \mathbf{q}), 30 \mu \mathrm{m}$ in other panels. Numbers below each bar represent the number of flies tested. Kruskal-Wallis test with Dunn's posttest, ${ }^{\star \star \star} P<0.001$, n.s. $P>0.05$. Error bars represent s.e.m. Male flies were used 
Supplementary Fig. 6), we found that the expression of SR in cells labeled by MyoIA-Gal4, Elav-Gal80 is essential for sleep regulation. We have examined the expression patterns only in the CNS, the gut, the genital, the fat body, and the oenocytes, therefore, although it is most likely that SR in the intestinal cells functions to regulate sleep, functions in other organs or cells could not be completely ruled out.

This is the first time that a gene has been found to function in the intestines to regulate sleep in any animal species. Why and how intestinal SR regulates sleep remains elusive. Sleep disorders have been found to be associated with gastrointestinal and metabolism pathology in human ${ }^{56}$ and animals ${ }^{57}$. The intestine is a tissue made up of a large variety of cell $s^{58}$ that could both sense the environment and communicate with the central nervous system. In mammals and flies, crosstalk between enteroendocrine cells and neurons through neuropeptide signaling have been identified to regulate processes such as energy homeostasis and development ${ }^{59,60}$, and the gut microbiome has been implicated in the regulation of behaviors such as locomotion and anxiety ${ }^{61-63}$. We have now demonstrated an essential role for an endogenous gene in the intestine in sleep regulation. How D-Ser produced in the intestine functions through the NMDAR to regulate sleep, and whether other cells, such as glia, participate in the circuit requires further studies. Our work should stimulate further investigations of whether $s r$ or other genes function in the gastrointestinal system to regulate sleep or other neuronal functions.

\section{Methods}

Fly lines and rearing conditions. All fly stocks were reared on standard corn meal at $25^{\circ} \mathrm{C}$ and $50 \%$ humidity on a 12:12 LD schedule unless otherwise noted. ElavGal4, Elav-Gal80, UAS-mCD8::GFP, UAS-StingerRed, LexAop-GFP were from the Bloomington Stock Center. MyoIA-Gal4 were generously provided by R. Xi (National Institute of Biological Science, Beijing). UAS-srRNAi (v110407) and UAS-Dicer (v60009) were from the Vienna Drosophila RNAi Center.

Generation of transgenic, knockout, and knockin flies. We generated UAS-SR, UAS-CG12338, and UAS-CG11236 flies by inserting the coding sequences of $C G 8129-R B, C G 12338-R A$, and CG11236-RA respectively into the PACU2 vector from the Jan lab at UCSF ${ }^{64}$, and then inserted the construct into attp 2 site. The coding sequences were amplified from the 1st strand cDNA made by the PrimeScript $^{\text {Ts }}$ II 1st strand cDNA synthesis kit (Takara, 6210A) from total RNA of $w t$ flies isolated with TRIzol reagent (Invitrogen).

We generated fly mutants with CRISPR/Cas9. A pSP6-2sNLS-spcas9 plasmid and a pMD19-T gRNA scaffold vector were obtained from Dr. R. Jiao ${ }^{65}$. After the sequence of single strand guide RNA (sgRNA) was designed, the corresponding DNA template was amplified from the pMD19-T gRNA scaffold vector, and then transcribed in vitro (Promega, P1320) to obtain the sgRNA. pSP6-2sNLS-spcas9 vector was linearized by restriction enzyme XbaI (New England BioLabs, USA, R0145), transcribed in vitro (Ambion, USA, AM1340), and added with Poly(A) (New England BioLabs, USA, M0276) to obtain spCas9 mRNA. To generate shmtes mutant, we injected one single strand guide RNA (sgRNA) and a spCas9 mRNA into Canton-S (CS) embryos to generate indel induced by site-directed cleavage in CG3011. F2 indel flies were identified by PCR and confirmed by sequencing. Mutants with a stop codon in the coding region of CG3011 were selected for further studies. To generate srko, CG12338ko, and CG11236ko flies, we injected two sgRNAs and a spCas9 mRNA into CS embryos. The target region between the two sgRNAs was deleted. F2 knock-out flies were identified by PCR and sequencing.

To obtain Gal4/LexA lines, we injected two sgRNA, spCas9 mRNA, and a donor plasmid into CS embryos. Gal4/LexA in the donor plasmid was integrated into specific sites of the genome through homologous recombination. A sgRNA and a spCas9 mRNA were used to improve the efficiency of homologous recombination. To construct the donor plasmid, two homologous arms were amplified by PCR from the fly genome using restriction enzyme-tailed PCR primers and the products were inserted into appropriate restriction sites in the pBlueScriptII vector. T2A-Gal4/LexAloxP-3P3-RFP-loxP sequence (constructed by Bowen Deng in the Lab) was inserted between the two homologous arms. T2A-Gal4/LexA was kept in-frame with the $5^{\prime}$ homologous arm. 3P3-RFP was used as a selection marker after injection. F2 flies with RFP observed in the eyes was selected and confirmed by PCR and sequencing (Supplementary Table 1). Sequences of the primers used for identification of the fly lines are presented in Supplementary Table 2.

Behavioral assays. Sleep analysis was performed in a video-based recording system. 5-8 days old flies were placed in $65 \mathrm{~mm} \times 5 \mathrm{~mm}$ tubes containing fly food.
Infrared LED lights were used to provide constant illumination, and videos were recorded by a camera with $704 \times 576$ resolution. Videos were taken at 5 frames/s. And 1 frame/s was extracted for fly tracing. The position of a fly was tracked by a program based on OpenCV. Briefly, flies were extracted by subtracting background which was updated for each frame in order to prevent environmental light shift. The center of a fly was calculated, the speed was defined as changes of the center from the previous frame to the current frame. Sleep was defined with more than 5 min bout of inactivity, sleep latency was defined as the time in minutes from the moment light was turned off to the onset of sleep ${ }^{16,17}$.

Arousal response was measured at ZT16 (4 h after lights off) under $1 \mathrm{~s}$ light stimulation (100-200 lux $)^{34}$. The percentage of flies that were aroused by light stimuli from sleep was calculated as arousal rate.

Sleep deprivation was performed by placing a silica gel holder with recording tubes horizontally into a holding box. The box was rotated clock-wise or counter clock-wise and bumped to plastic stoppers under the control of a servo motor. The box was rotated continuously for 9 times during each episode, and the setup was activated every $3 \mathrm{~min}$ for $12 \mathrm{~h}$ during the night. Sleep was completely deprived as confirmed by DAM recording during deprivation ${ }^{66}$. Because sleep homeostasis is more significant in females than that in males $^{67}$, we used females to examine sleep homeostasis.

For analysis of circadian rhythm, flies were treated and recorded in the same condition as the sleep assay, except that the experiments were performed in DD. Activity was measured for 5-8 days and calculated in Actogram ${ }^{68}$. The period length was calculated by Chi-square method.

Drug treatment. L-Ser (S0035) and D-Ser (S0033) were from Tokyo Chemical Industry and mixed in the $5 \%$ sucrose and $2 \%$ agar medium. Flies were maintained on food containing $2.9 \mathrm{~g} / \mathrm{L} \mathrm{L}$-Ser, D-Der (treatment group) or no Ser (control group) after eclosion for 3 days before being transferred to the recording tube with the same medium.

Immunohistochemistry and confocal imaging. Flies were anesthetized and dissected in cold phosphate-buffered saline (PBS). Brains were fixed in $4 \%$ paraformaldehyde (weight/volume) for $1 \mathrm{~h}$ at room temperature (RT), washed in PBST (PBS containing $0.2 \%$ Triton $\mathrm{X}-100$, vol/vol) for $10 \mathrm{~min}$ three times, blocked in PBSTS (PBS containing 2\% Triton X-100, 10\% normal goat serum, vol/vol) for $12 \mathrm{~h}$ at $4{ }^{\circ} \mathrm{C}$, incubated with primary antibodies in the dilution buffer (PBS containing $0.25 \%$ Triton X-100, $1 \%$ normal goat serum, vol/vol) for $12 \mathrm{~h}$ at $4{ }^{\circ} \mathrm{C}$, and washed with the washing buffer (PBS containing $1 \%$ Triton X-100, vol/vol, $3 \% \mathrm{NaCl}, \mathrm{g} / \mathrm{ml}$ ) for $10 \mathrm{~min}$ three times. Brains were then incubated with secondary antibodies in the dilution buffer for $12 \mathrm{~h}$ at $4{ }^{\circ} \mathrm{C}$ in darkness, and washed three times in the washing buffer for $10 \mathrm{~min}$ each. In the case of the third antibody, brains were further incubated with third antibodies at $4{ }^{\circ} \mathrm{C}$ overnight and washed. Finally, brains were mounted in Focusclear (Cell Explorer Labs, FC-101) and imaged on Zeiss LSM710 confocal microscope.

The intestines, after dissection, were fixed with $4 \%$ paraformaldehyde for $0.5 \mathrm{~h}$, followed by $30 \mathrm{~s}$ in heptanes and methanol $(1: 1$, vol/vol), washed with $100 \%$ methanol for 5 min twice, and washed with PBST for 10 min three times, and immunostained for GFP as described in the previous paragraph. Images were processed by Zeiss software and Imaris (bitplane) for 3D reconstruction.

Chicken anti-GFP (1:1000) (Abcam Cat\# 13970; RRID:AB_300798), mouse anti-Bruchpilot (1:40) (DSHB Cat\# 2314866, nc82; RRID: AB_2314866) were used as primary antibodies with AlexaFluor488 anti-chicken (1:500) (Life Technologies Cat\# A11039; RRID:AB_2534096) and AlexaFluor633 anti-mouse (1:500) (Life Technologies Cat\# A21052; RRID: AB_141459) being used as respective secondary antibodies. Mouse 4F3 anti-DLG (1:50) (DSHB Cat\# 4F3 anti-discs large; RRID: AB_528203) was used as primary antibody with biotin-conjugated goat anti-mouse (1:200) (Invitrogen Cat\# B2763; RRID: AB_2536430) being the secondary antibody and AlexaFluor635 streptavidin (1:500) (Invitrogen Cat\# S32364) being the third antibody.

Quantitative PCR. Total RNA was extracted from $\sim 60$ flies aged 5-8 days using TRIzol reagent (Invitrogen) and then reverse transcripted by the PrimeScript ${ }^{\mathrm{TM}} \mathrm{RT}$ Master Mix kit (Takara, RR036A). Quantitative PCR analysis was then performed using TransStart Top Green qPCR SuperMix kit (TransGen, AQ131-03) in the Applied Biosystems 7900HT Fast-Time PCR system. The sequences of primers used to detect shmt and actin42a (endogenous control) RNA are as follows: shmt-F: 5'-CAGCCGTTTACAAAGACATGCA-3

shmt-R: 5'-GAATGGCGTTGGTGATGGTT-3' act42a-F: 5'-CTCCTACATATTTCCATAAAAGATCCAA-3' act42a-R: 5'-GCCGACAATAGAAGGAAAAACTG-3'

Statistics. All statistical analyses were carried out with Prism 5 (GraphPad Software). Fisher's exact test was used to compare arousal rates. Mann-Whitney test was used to compare two columns of data. Kruskal-Wallis test followed by Dunn's posttest was used to compare multiple columns of data. Two-way ANOVA followed by Bonferroni post-tests was used to compare drug rescue effects. Additional Mann-Whitney test was used to compare mutants with wt flies under different 
treatments. Statistical significance is denoted by asterisks: ${ }^{*} P<0.05,{ }^{* *} P<0.01$, ${ }_{* * *} P<0.001$.

\section{Data availability}

The data that support the findings of this study are available upon reasonable request.

\section{Code availability}

Code used for tracing the fly were conducted in $\mathrm{C}++$, and further analyses were conducted in Matlab. All the code are available upon reasonable request.

Received: 19 June 2018 Accepted: 14 March 2019

Published online: 07 May 2019

\section{References}

1. Corrigan, J. J. D-amino acids in animals. Science 164, 142-149 (1969).

2. Nagata, Y., Konno, R., Yasumura, Y. \& Akino, T. Involvement of D-amino acid oxidase in elimination of free D-amino acids in mice. Biochem. J. 257, 291-292 (1989).

3. Hashimoto, A. et al. The presence of free D-serine in rat brain. FEBS Lett. 296, 33-36 (1992).

4. Nagata, Y. Involvement of D-amino acid oxidase in elimination of D-serine in mouse brain. Experientia 48, 753-755 (1992).

5. Fadda, E., Danysz, W., Wroblewski, J. T. \& Costa, E. Glycine and D-serine increase the affinity of $\mathrm{N}$-methyl-D-aspartate sensitive glutamate binding sites in rat brain synaptic membranes. Neuropharmacology 27, 1183-1185 (1988).

6. Kleckner, N. W. \& Dingledine, R. Requirement for glycine in activation of NMDA-receptors expressed in Xenopus oocytes. Science 241, 835-837 (1988).

7. Wolosker, H. et al. Purification of serine racemase: biosynthesis of the neuromodulator D-serine. Proc. Natl. Acad. Sci. USA 96, 721-725 (1999)

8. De Miranda, J., Panizzutti, R., Foltyn, V. N. \& Wolosker, H. Cofactors of serine racemase that physiologically stimulate the synthesis of the N-methylD-aspartate (NMDA) receptor coagonist D-serine. Proc. Natl. Acad. Sci. USA 99, 14542-14547 (2002).

9. Hashimoto, A., Nishikawa, T., Oka, T. \& Takahashi, K. Endogenous D-serine in rat brain: $\mathrm{N}$-methyl-D-aspartate receptor-related distribution and aging. J. Neurochem. 60, 783-786 (1993).

10. Schell, M. J., Brady, R. O., Molliver, M. E. \& Snyder, S. H. D-serine as a neuromodulator: regional and developmental localizations in rat brain glia resemble NMDA receptors. J. Neurosci. 17, 1604-1615 (1997).

11. Matsui, T. et al. Functional comparison of D-serine and glycine in rodents: the effect on cloned NMDA receptors and the extracellular concentration. J. Neurochem. 65, 454-458 (1995).

12. Priestley, T. et al. Pharmacological properties of recombinant human Nmethyl-D-aspartate receptors comprising NR1a/NR2A and NR1a/NR2B subunit assemblies expressed in permanently transfected mouse fibroblast cells. Mol. Pharmacol. 48, 841-848 (1995).

13. Mothet, J. P. et al. D-serine is an endogenous ligand for the glycine site of the N-methyl-D-aspartate receptor. Proc. Natl. Acad. Sci. USA 97, 4926-4931 (2000).

14. Papouin, T. et al. Synaptic and extrasynaptic NMDA receptors are gated by different endogenous coagonists. Cell 150, 633-646 (2012).

15. Borbély, A. A., Daan, S., Wirz-Justice, A. \& Deboer, T. The two-process model of sleep regulation: a reappraisal. J. Sleep Res. 25, 131-143 (2016).

16. Hendricks, J. C. et al. Rest in Drosophila is a sleep-like state. Neuron 25, 129-138 (2000).

17. Shaw, P. J., Cirelli, C., Greenspan, R. J. \& Tononi, G. Correlates of sleep and waking in Drosophila melanogaster. Science 287, 1834-1837 (2000).

18. Cirelli, C. The genetic and molecular regulation of sleep: from fruit flies to humans. Nat. Rev. Neurosci. 10, 549-560 (2009).

19. Crocker, A. \& Sehgal, A. Genetic analysis of sleep. Genes Dev. 24, 1220-1235 (2010).

20. Axelrod, S., Saez, L. \& Young, M. W. Studying circadian rhythm and sleep using genetic screens in Drosophila. Methods Enzymol. 551, 3-27 (2015)

21. Artiushin, G. \& Sehgal, A. The Drosophila circuitry of sleep-wake regulation. Curr. Opin. Neurobiol. 44, 243-250 (2017).

22. Tomita, J., Ueno, T., Mitsuyoshi, M., Kume, S. \& Kume, K. The NMDA receptor promotes sleep in the fruit fly, Drosophila melanogaster. PLoS One 10, e0128101 (2015)

23. Liu, S., Liu, Q., Tabuchi, M. \& Wu, M. N. Sleep drive is encoded by neural plastic changes in a dedicated circuit. Cell 165, 1347-1360 (2016).

24. Papouin, T., Dunphy, J. M., Tolman, M., Dineley, K. T. \& Haydon, P. G. Septal cholinergic neuromodulation tunes the astrocyte-dependent gating of hippocampal NMDA receptors to wakefulness. Neuron 94, 840-854 (2017).
25. Narkewicz, M. R. et al. Serine and glycine metabolism in hepatocytes from mid gestation fetal lambs. Pediatr. Res. 39, 1085-1090 (1996).

26. Tibbetts, A. S. \& Appling, D. R. Compartmentalization of mammalian folatemediated one-carbon metabolism. Annu. Rev. Nutr. 30, 57-81 (2010)

27. Zhou, C., Rao, Y. \& Rao, Y. A subset of octopaminergic neurons are important for Drosophila aggression. Nat. Neurosci. 11, 1059-1067 (2008).

28. Inoue, R., Hashimoto, K., Harai, T. \& Mori, H. NMDA- and betaamyloid1-42-induced neurotoxicity is attenuated in serine racemase knockout mice. J. Neurosci. 28, 14486-14491 (2008)

29. Basu, A. C. et al. Targeted disruption of serine racemase affects glutamatergic neurotransmission and behavior. Mol. Psychiatry 14, 719-727 (2009).

30. Labrie, V. et al. Serine racemase is associated with schizophrenia susceptibility in humans and in a mouse model. Hum. Mol. Genet. 18, 3227-3243 (2009).

31. Kong, E. C. et al. Ethanol-regulated genes that contribute to ethanol sensitivity and rapid tolerance in Drosophila. Alcohol. Clin. Exp. Res. 34, 302-316 (2010).

32. Faville, R., Kottler, B., Goodhill, G. J., Shaw, P. J. \& van Swinderen, B. How deeply does your mutant sleep? Probing arousal to better understand sleep defects in Drosophila. Sci. Rep. 5, 8454 (2015).

33. Lebestky, T. et al. Two different forms of arousal in Drosophila are oppositely regulated by the dopamine D1 receptor ortholog DopR via distinct neural circuits. Neuron 64, 522-536 (2009)

34. Kayser, M. S., Yue, Z. \& Sehgal, A. A critical period of sleep for development of courtship circuitry and behavior in Drosophila. Science 344, 269-274 (2014).

35. Wolosker, H. \& Mori, H. Serine racemase: an unconventional enzyme for an unconventional transmitter. Amino Acids 43, 1895-1904 (2012).

36. Pollegioni, L., Piubelli, L., Sacchi, S., Pilone, M. S. \& Molla, G. Physiological functions of D-amino acid oxidases: from yeast to humans. Cell. Mol. Life Sci. 64, 1373-1394 (2007).

37. Ultsch, A., Schuster, C. M., Laube, B., Betz, H. \& Schmitt, B. Glutamate receptors of Drosophila melanogaster. Primary structure of a putative NMDA receptor protein expressed in the head of the adult fly. FEBS Lett. 324, 171-177 (1993)

38. Jiang, H. \& Edgar, B. A. EGFR signaling regulates the proliferation of Drosophila adult midgut progenitors. Development 136, 483-493 (2009).

39. Colwell, C. S., Foster, R. G. \& Menaker, M. NMDA receptor antagonists block the effects of light on circadian behavior in the mouse. Brain Res. 554, 105-110 (1991)

40. Colwell, C. S. \& Menaker, M. NMDA as well as non-NMDA receptor antagonists can prevent the phase-shifting effects of light on the circadian system of the Golden Hamster. J. Biol. Rhythms 7, 125-136 (1992).

41. Kékesi, K. A., Dobolyi, A., Salfay, O., Nyitrai, G. \& Juhász, G. Slow wave sleep is accompanied by release of certain amino acids in the thalamus of cats. Neuroreport 8, 1183-1186 (1997).

42. Lockridge, A. et al. Timing-dependent reduction in ethanol sedation and drinking preference by NMDA receptor co-agonist D-serine. Alcohol 46, 389-400 (2012)

43. Almond, S. L. et al. Behavioral and biochemical characterization of a mutant mouse strain lacking D-amino acid oxidase activity and its implications for schizophrenia. Mol. Cell. Neurosci. 32, 324-334 (2006).

44. Pritchett, D. et al. d-amino acid oxidase knockout $(\mathrm{Dao}(-/-))$ mice show enhanced short-term memory performance and heightened anxiety, but no sleep or circadian rhythm disruption. Eur. J. Neurosci. 41, 1167-1179 (2015)

45. Labrie, V. et al. Genetic inactivation of D-amino acid oxidase enhances extinction and reversal learning in mice. Learn. Mem. 16, 28-37 (2009).

46. Mori, H. \& Inoue, R. Serine racemase knockout mice. Chem. Biodivers. 7, 1573-1578 (2010)

47. Wolosker, H., Blackshaw, S. \& Snyder, S. H. Serine racemase: a glial enzyme synthesizing D-serine to regulate glutamate-N-methyl-D-aspartate neurotransmission. Proc. Natl. Acad. Sci. USA 96, 13409-13414 (1999).

48. Schell, M. J., Molliver, M. E. \& Snyder, S. H. D-serine, an endogenous synaptic modulator: localization to astrocytes and glutamate-stimulated release. Proc. Natl. Acad. Sci. USA 92, 3948-3952 (1995).

49. Mothet, J. P. et al. Glutamate receptor activation triggers a calcium-dependent and SNARE protein-dependent release of the gliotransmitter D-serine. Proc. Natl. Acad. Sci. USA 102, 5606-5611 (2005).

50. Martineau, M. et al. Storage and uptake of D-serine into astrocytic synapticlike vesicles specify gliotransmission. J. Neurosci. 33, 3413-3423 (2013).

51. Kang, N. et al. Astrocytes release D-serine by a large vesicle. Neuroscience $\mathbf{2 4 0}$, 243-257 (2013).

52. Kartvelishvily, E., Shleper, M., Balan, L., Dumin, E. \& Wolosker, H. Neuronderived D-serine release provides a novel means to activate N-methyl-Daspartate receptors. J. Biol. Chem. 281, 14151-14162 (2006).

53. Miya, K. et al. Serine racemase is predominantly localized in neurons in mouse brain. J. Comp. Neurol. 510, 641-654 (2008).

54. Rosenberg, D. et al. Neuronal release of D-serine: a physiological pathway controlling extracellular D-serine concentration. FASEB J. 24, 2951-2961 (2010). 
55. Benneyworth, M. A., Li, Y., Basu, A. C., Bolshakov, V. Y. \& Coyle, J. T. Cell selective conditional null mutations of serine racemase demonstrate a predominate localization in cortical glutamatergic neurons. Cell. Mol. Neurobiol. 32, 613-624 (2012).

56. Hasler, G. et al. The association between short sleep duration and obesity in young adults: a 13-year prospective study. Sleep 27, 661-666 (2004).

57. Everson, C. A. \& Wehr, T. A. Nutritional and metabolic adaptations to prolonged sleep deprivation in the rat. Am. J. Physiol. 264, R376-R387 (1993).

58. Haber, A. L. et al. A single-cell survey of the small intestinal epithelium. Nature 551, 333-339 (2017).

59. Droujinine, I. A. \& Perrimon, N. Interorgan communication pathways in physiology: focus on Drosophila. Annu. Rev. Genet. 50, 539-570 (2016).

60. Liu, Q. \& Jin, L. H. Organ-to-organ communication: a Drosophila gastrointestinal tract perspective. Front. Cell Dev. Biol. 5, 29 (2017)

61. Collins, S. M., Surette, M. \& Bercik, P. The interplay between the intestinal microbiota and the brain. Nat. Rev. Microbiol. 10, 735-742 (2012)

62. Sharon, G., Sampson, T. R., Geschwind, D. H. \& Mazmanian, S. K. The central nervous system and the gut microbiome. Cell 167, 915-932 (2016).

63. Schretter, C. E. et al. A gut microbial factor modulates locomotor behaviour in Drosophila. Nature 563, 402-406 (2018).

64. Han, C., Jan, L. Y. \& Jan, Y.-N. Enhancer-driven membrane markers for analysis of nonautonomous mechanisms reveal neuron-glia interactions in Drosophila. Proc. Natl. Acad. Sci. USA 108, 9673-9678 (2011).

65. Yu, Z. et al. Highly efficient genome modifications mediated by CRISPR/Cas 9 in Drosophila. Genetics 195, 289-291 (2013).

66. Qian, Y. et al. Sleep homeostasis regulated by $5 \mathrm{HT} 2 \mathrm{~b}$ receptor in a small subset of neurons in the dorsal fan-shaped body of Drosophila. eLife 6, e26519 (2017).

67. Huber, R. et al. Sleep homeostasis in Drosophila melanogaster. Sleep 27, 628-639 (2004).

68. Schmid, B., Helfrich-Förster, C. \& Yoshii, T. A new ImageJ plug-in 'ActogramJ' for chronobiological analyses. J. Biol. Rhythms 26, 464-467 (2011).

\section{Acknowledgements}

The authors are grateful to Guang Yang for assistance with imaging, to Pingping Yan for fly rearing, to Peking-Tsinghua Center for Life Sciences, the Beijing Advanced Innovation Center for Genomics, the Beijing Commission of Science and Technology
(Z151100003915121 to W.Z.), and the National Natural Science Foundation of China (Project 31421003 to Y.R.) for grant support.

\section{Author contributions}

X.D., E.Z., and Y.R. conceived and directed the study. X.D. and E.Z. designed and made Drosophila lines. E.Z. and X.D. performed behavioral experiments and data analysis. W.Y. and E.Z. wrote computer programs. E.Z., X.D., and Y.R. wrote the manuscript. X.Z and W.Z. contributed to experiments.

\section{Additional information}

Supplementary Information accompanies this paper at https://doi.org/10.1038/s41467019-09544-9.

Competing interests: The authors declare no competing interests.

Reprints and permission information is available online at http://npg.nature.com/ reprintsandpermissions/

Journal peer review information: Nature Communications would like to thank the anonymous reviewers for their contribution to the peer review of this work.

Publisher's note: Springer Nature remains neutral with regard to jurisdictional claims in published maps and institutional affiliations.

(i) Open Access This article is licensed under a Creative Commons Attribution 4.0 International License, which permits use, sharing, adaptation, distribution and reproduction in any medium or format, as long as you give appropriate credit to the original author(s) and the source, provide a link to the Creative Commons license, and indicate if changes were made. The images or other third party material in this article are included in the article's Creative Commons license, unless indicated otherwise in a credit line to the material. If material is not included in the article's Creative Commons license and your intended use is not permitted by statutory regulation or exceeds the permitted use, you will need to obtain permission directly from the copyright holder. To view a copy of this license, visit http://creativecommons.org/ licenses/by/4.0/.

(C) The Author(s) 2019 